\title{
Phosphatidylinositol 3-kinase p85 $\alpha$ regulatory subunit gene PIK3R1 haplotype is associated with body fat and serum leptin in a female twin population
}

\author{
Y. Jamshidi $\cdot$ H. Snieder $\cdot$ X. Wang $\cdot$ M. J. Pavitt • \\ T. D. Spector • N. D. Carter $\cdot$ S. D. O'Dell
}

Received: 6 April 2006 / Accepted: 18 June 2006 / Published online: 20 September 2006

(C) Springer-Verlag 2006

\begin{abstract}
Aims/hypothesis Phosphatidylinositol 3-kinase (PI3K) couples the leptin and insulin signalling pathways via the insulin receptor substrates IRS1 and IRS2. Hence, defective activation of PI3K could be a novel mechanism of peripheral leptin or insulin resistance. We investigated associations of tagging single-nucleotide polymorphisms (tSNPs) in the PI3K p85 $\alpha$ regulatory subunit gene PIK3RI with anthropometry, leptin, body fat and insulin sensitivity in a female twin population of European extraction.

Materials and methods Eight tSNPs were genotyped in 2,778 women (mean age $47.4 \pm 12.5$ years) from the $\mathrm{St}$ Thomas' UK Adult Twin Registry (Twins UK).

Results SNP rs1550805 was associated with serum leptin $(p=0.028)$, BMI $(p=0.025)$, weight $(p=0.019)$, total fat
\end{abstract}

Electronic supplementary material Supplementary material is available in the online version of this article at http://dx.doi.org/ $10.1007 / \mathrm{s} 00125-006-0388-\mathrm{z}$ and is accessible to authorised users.

Y. Jamshidi · M. J. Pavitt · N. D. Carter

Department of Clinical Developmental Sciences,

St George's, University of London,

London, UK

H. Snieder $\cdot X$. Wang

Georgia Prevention Institute, Department of Pediatrics,

Medical College of Georgia,

Augusta, GA, USA

H. Snieder $\cdot$ T. D. Spector

Twin Research and Genetic Epidemiology Unit,

St Thomas' Campus, King's College London,

London, UK

S. D. O’Dell ( $\square)$

Nutrition Food and Health Research Centre,

King's College London,

Franklin-Wilkins Building, 150 Stamford Street,

London SE1 9NH, UK

e-mail: sandra.o'dell@kcl.ac.uk $(p=0.004)$, total fat percentage $(p=0.002)$, waist circumference $(p=0.025)$, central fat $(p=0.005)$ and central fat percentage $(p=0.005)$. SNPs rs7713645 and rs7709243 were associated with BMI ( $p=0.020$ and $p=0.029$, respectively), rs7709243 with weight, total and central fat ( $p=0.026, p=0.031$ and $p=0.023$, respectively) and both SNPs with fasting glucose $(p=0.003$ and $p=0.001$, respectively) and glucose 2 -h post OGTT ( $p=0.023$ and $p=0.007$, respectively). Subjects with haplotype 222 (frequency $7.2 \%)$ showed higher serum leptin concentration $(p=0.007)$ and body fat measures $(p \leq 0.001$ for all), and those with haplotype 221 (frequency 38.7\%) showed higher fasting and 2-h glucose ( $p=0.035$ and $p=0.021$, respectively) compared with subjects with the most common haplotype, 111 (frequency 45.5\%).

Conclusions/interpretation Association of the PIK3R1 SNP rs1550805 with serum leptin and body fat may reflect a diminished ability of PI3K to signal via IRS1 or IRS2 in response to leptin.

Keywords Cytokines · Epidemiology · Genetics · Obesity · Weight regulation

$\begin{array}{ll}\text { Abbreviations } \\ \mathrm{D}^{\prime} & \text { pairwise linkage disequilibrium statistic } \\ \text { ESM } & \text { Electronic Supplementary Material } \\ \text { GEE } & \text { generalised estimating equations } \\ \text { JAK2 } & \text { Janus activating kinase 2 } \\ \text { LD } & \text { linkage disequilibrium } \\ \text { MAF } & \text { minor allele frequency } \\ \text { NCBI } & \text { National Center for Biotechnology Information } \\ & \text { (USA) } \\ \text { PI3K } & \text { phosphatidylinositol 3-kinase } \\ \text { PIK3R1 } & \text { PI3K p85 } \alpha \text { regulatory subunit gene } \\ \text { PKB } & \text { protein kinase B } \\ \text { SiM } & \text { insulin sensitivity measure }\end{array}$


SNP single-nucleotide polymorphism

tSNP tagging single-nucleotide polymorphism

\section{Introduction}

In the hypothalamus, insulin and leptin function as afferent adiposity signals, which are important for the regulation of body fat stores and glucose metabolism [1]. Insulin induces tyrosine phosphorylation of the insulin receptor, whose intrinsic kinase activity phosphorylates insulin receptor substrates IRS1 and IRS2. Binding of activated IRS1 and IRS 2 to the $\mathrm{p} 85 \alpha$ regulatory subunit of phosphatidylinositol 3-kinase (PI3K) then leads to association with the p110 catalytic subunit and activation of downstream targets via protein kinase B (PKB) [2]. Binding of leptin to hypothalamic receptors, inducing tyrosine-phosphorylation of Janus activating kinase 2 (JAK2) and signal transducer and activator of transcription 3 (STAT3), is paralleled by a comparable increase in PI3K activity associated with IRS2 [3] and to a lesser extent with IRS1 [4-6]. Hence IRS1 and IRS2 represent convergence points in the two hypothalamic signalling pathways and cross-talk could explain the overlapping activities of these two hormones [3].

Overweight individuals have high circulating levels of leptin, implying central resistance to its effect as an adiposity factor [7]. It has been established that pharmacological inhibition of PI3K activity in the hypothalamus inhibits the effects of leptin on food intake [3, 8], suggesting that defective activation of hypothalamic PI3K may play a role in central leptin resistance. Recently, leptin has been shown to increase PI3K activity in the liver, leading to lowering of hepatic triglyceride levels in lean rats, but not in obese animals on a high-fat diet [9]. Hence, defective activation of PI3K could also be a novel mechanism of peripheral leptin resistance. Direct interaction between PI3K and membrane vesicles leads to mobilisation of GLUT4 glucose transporters in response to insulin stimulation in adipose, muscle and liver cells [10], so it is possible that defective PI3K activity may also contribute to peripheral insulin resistance.

The PI3K regulatory subunit isoforms $\mathrm{p} 85 \alpha, \mathrm{p} 55 \alpha$ and $\mathrm{p} 50 \alpha$, which are generated from the same PIK $3 R 1$ gene by alternative splicing [11], negatively modulate catalytic activity to different extents [12], the strongest effect being shown by $\mathrm{p} 85 \alpha$ [13]. The $\mathrm{p} 85 \alpha$ protein includes two SH2 domains, which bind to specific phosphotyrosines of IRS1 and IRS2 [14] and are preserved in the p55 $\alpha$ and p $50 \alpha$ variants [15]. Three amino acid substitutions have been identified in PIK3R1, Met326Ile (rs3730089) [16], Arg409Gln in one insulin-resistant subject [17], and an unvalidated single-nucleotide polymorphism (SNP),
Ala440Asp (rs3730091). The first was associated with alterations in glucose/insulin homeostasis in one study [16] but not in a larger sample [18]. One other study [19] found an association between intronic SNP IVS4+82 and type 2 diabetes.

Aside from Met326Ile and IVS4+82, there are no other studies of association of PIK3R1 variants with variables characterising the action of leptin or insulin. Rather than focusing on one or two functional SNPs, which are often difficult to replicate, examination on a gene-wide level is preferable, common variants within a candidate gene being considered jointly through the selection of a minimal set of tagging SNPs (tSNPs) [20]. These effectively capture information about the common variants by taking into account patterns of linkage disequilibrium (LD) across the gene [21, 22]. We selected a set of eight tSNPs in PIK3RI and tested their association with a range of variables characterising leptin, body fat and insulin sensitivity in a large population of female twins $(n=2,778$; mean \pm SD age $47.4 \pm 12.5$ years), approximately double the size of the larger study of Hansen et al. [18]. SNPs were tested individually and as haplotypes.

\section{Subjects and methods}

Study design

The Twins UK Registry comprises unselected, mostly female volunteers ascertained from the general population through national media campaigns in the UK [23]. The study cohort included 2,778 subjects (423 monozygotic pairs, 940 dizygotic pairs and 52 singletons) with available leptin data. No difference was observed in the distributions of age and menopausal status between subjects with and without leptin data $(n=362)$. The number of individuals in the study cohort with data on other phenotypic variables is shown in Table 1.

Means and ranges of quantitative phenotypes in Twins UK are similar to an age-matched sample of the UK female population [24]. Informed consent was obtained from participants before they entered the study, and the study was approved by the local research ethics committee.

\section{Zygosity, body composition and biochemical analyses}

Zygosity was determined by a standardised questionnaire and confirmed by DNA fingerprinting. Height was measured to the nearest $0.5 \mathrm{~cm}$ using a wall-mounted stadiometer. Weight (light clothing only) was measured to the nearest $0.1 \mathrm{~kg}$ using digital scales. BMI was used as a measure of general adiposity and calculated as weight divided by height squared $\left(\mathrm{kg} / \mathrm{m}^{2}\right)$. Waist circumference 
Table 1 General characteristics of Twins UK subjects

\begin{tabular}{lll}
\hline Variable & $n$ & Mean $\pm \mathrm{SD}$ \\
\hline Age (years) & $2,778^{\mathrm{a}}$ & $47.4 \pm 12.5$ \\
Postmenopausal (\%) & 2,455 & 47.7 \\
Obesity-related variables & & \\
Leptin $(\mathrm{ng} / \mathrm{ml})$ & 2,778 & $16.5 \pm 12.0$ \\
BMI $\left(\mathrm{kg} / \mathrm{m}^{2}\right)$ & 2,762 & $24.8 \pm 4.4$ \\
Weight $(\mathrm{kg})$ & 2,763 & $65.3 \pm 11.8$ \\
Waist circumference $(\mathrm{cm})$ & 2,711 & $78.4 \pm 10.2$ \\
Total fat $(\mathrm{kg})$ & 2,722 & $23.4 \pm 8.8$ \\
Total fat $(\%)$ & 2,681 & $35.6 \pm 8.0$ \\
Central fat $(\mathrm{kg})$ & 2,699 & $1.33 \pm 0.73$ \\
Central fat $(\%)$ & 2,699 & $31.2 \pm 11.5$ \\
Insulin sensitivity & & \\
Fasting glucose $(\mathrm{mmol} / \mathrm{l})$ & 2,318 & $4.49 \pm 0.55$ \\
Fasting insulin $\left(\mu \mathrm{U} / \mathrm{ml}^{\mathrm{b}}\right)$ & 1,986 & $6.97 \pm 5.96$ \\
2-h glucose $(\mathrm{mmol} / \mathrm{l})$ & 739 & $5.18 \pm 1.10$ \\
2-h insulin $\left(\mu \mathrm{U} / \mathrm{ml}^{2}\right)$ & 739 & $34.2 \pm 25.4$ \\
SiM $\left(10^{8} \mu \mathrm{U}^{-1} \mathrm{mmol}{ }^{-1} \mathrm{l}^{-1}\right)$ & 739 & $88.5 \pm 68.8$ \\
\hline
\end{tabular}

${ }^{\mathrm{a}}$ Number of subjects with leptin data and genotype data on at least one SNP; includes 423 monozygotic pairs, 940 dizygotic pairs and 52 singletons

${ }^{\mathrm{b}}$ Non-fasting subjects, patients with either type 1 or type 2 diabetes, patients on any glucose-lowering drugs, and subjects with fasting glucose $>7.8 \mathrm{mmol} / \mathrm{l}$ or 2 -h glucose $>11.1 \mathrm{mmol} / \mathrm{l}$ were all excluded

(cm) was measured at the level midway between the lower rib margin and the iliac crest. Body composition was measured by dual emission X-ray absorptiometry (Hologic QDR-2000, Vertec, Waltham, MA, USA). Serum leptin concentration was determined after an overnight fast using a radioimmunoassay (Linco Research, St Louis, MO, USA). Fasting insulin was measured by immunoassay (Abbott Laboratories, Maidenhead, UK) and glucose was measured on an Ektachem 700 multichannel analyser using an enzymatic colorimetric slide assay (Johnson and Johnson Clinical Diagnostic Systems, Amersham, UK). A subsample of approximately 739 subjects, representing unselected female twins from the general population, underwent an OGTT, for which glucose and insulin levels were measured before and $2 \mathrm{~h}$ after a 75 -g oral glucose load.

\section{Genotyping for SNP validation and tSNP selection}

We used the National Center for Biotechnology Information (USA) (NCBI; http://www.ncbi.nlm.nih.gov/SNP/) and CHIP Bioinformatics (http://www.snpperchip.org) databases to choose SNPs for validation and tSNP selection in our cohort. Information from the HapMap (http://www. hapmap.org) for PIK3RI was not available at the time of SNP selection. Thirteen SNPs validated in the database populations were genotyped in 94 unrelated subjects from the Twins UK cohort for tSNP selection. PCR primers and conditions are given in Electronic Supplementary Material (ESM) Table 1. This sample size is three times that suggested by one permutation study [25], which indicates that genotyping 25-32 unphased individuals is sufficient to select tSNPs. The rs numbers and relative positions of the 13 SNPs are shown in Fig. 1.

Genotyping in cohorts

Eight tSNPs (rs706713, rs7713645, rs7709243, rs251406, rs40318, rs1550805, rs831125 and rs3730089) were selected and genotyped in the complete Twins UK cohort by Pyrosequencing (Biotage, Uppsala, Sweden). Genotyping accuracy, as assessed by inclusion of duplicates (pairs of monozygotic twins) in the arrays, was $98 \%$ and negative controls (water blanks) were included on each plate. Genotyping success rate for each tSNP varied between 77.2 and $93.8 \%$. Primers and PCR conditions for SNP genotyping in the full cohort by Pyrosequencing are given in ESM Table 2.

Statistical analysis

The main purposes of our analyses were to select a set of tSNPs representing the common variants in PIK3R1 and to test the effects of these tSNPs, individually and/or as haplotypes, on a range of variables, including serum leptin, measures of body fat and insulin sensitivity.

We used the htSNP2 program developed by Chapman et al. [21] to identify the optimal subset of tSNPs, which were selected in such a way that the allele frequencies of the remaining (non-tagging SNPs) could be predicted well. A series of regression equations were calculated for which the predictive efficiency was assessed in terms of $R_{L}^{2}$, which measures the proportion of variance of each remaining SNP explained by regression on the tSNP alleles (locus-based scoring). htSNP2 was used to select a tSNP set that predicted remaining SNPs with a minimum $R_{L}^{2}$ of 0.80 , as recommended by Chapman et al. [21]. Pairwise LD coefficients in the tSNP test sample were calculated using GOLD (available from http://www.sph.umich.edu/csg/abe casis/GOLD/, last accessed in July 2006) and are reported as the pairwise linkage disequilibrium statistic $\left(\mathrm{D}^{\prime}\right)$ and $r^{2}$ [26].

Preliminary association analyses were performed using STATA 8 (StataCorp, College Station, TX, USA). Phenotypes significantly $(p<0.05)$ deviating from normal were log-transformed to obtain normal distributions prior to analysis. Hardy-Weinberg equilibrium was tested with a $\chi^{2}$ test with one degree of freedom in one twin of each pair chosen at random to prevent inflated significance. Association analyses were performed using generalised estimating equations (GEE) [27], a method that allows for the 


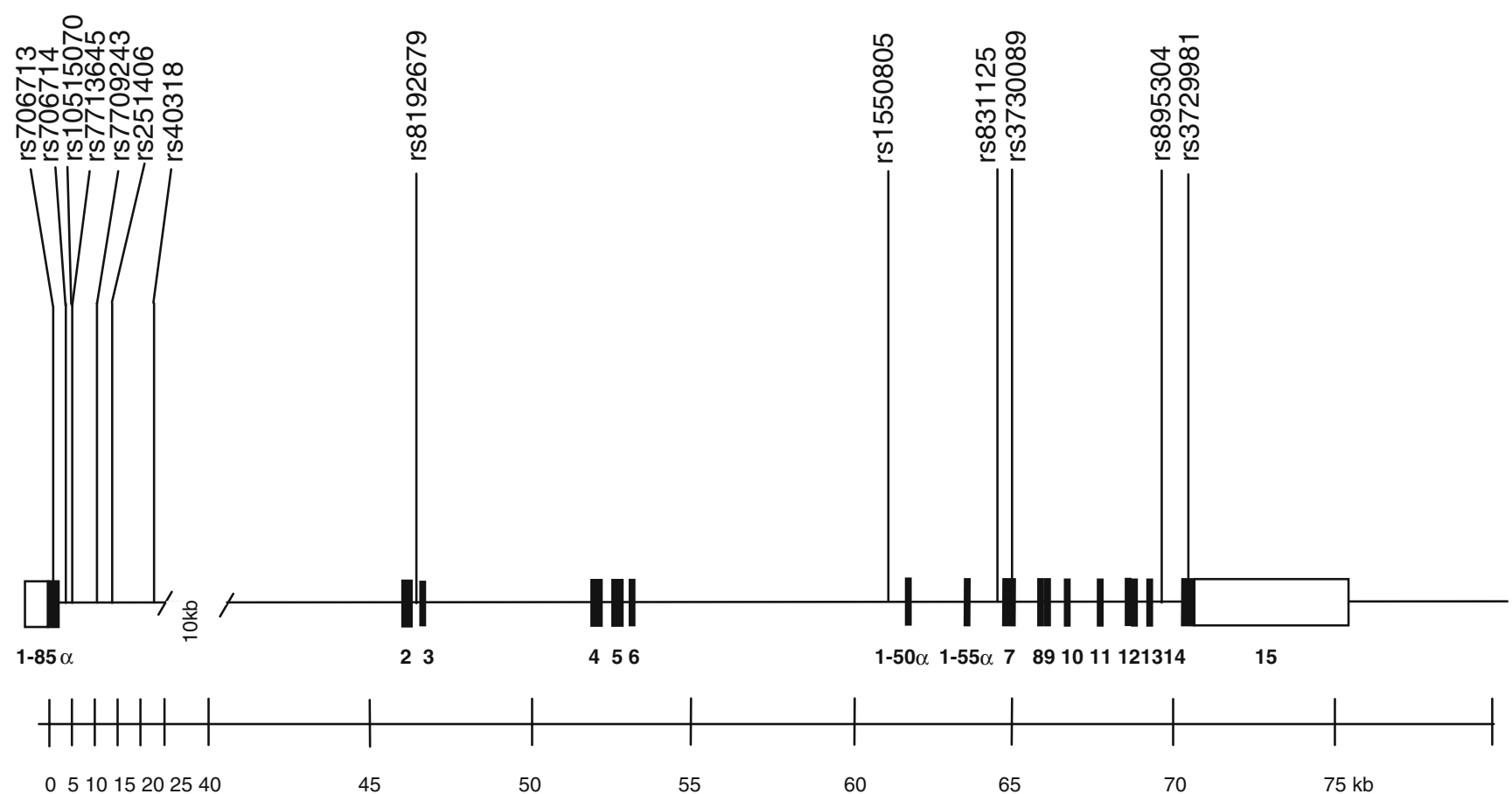

Fig. 1 Genomic map of PIK3R1 gene with rs numbers and locations of the 13 validated SNPs. Boxes represent exons. Solid boxes represent translated regions and open boxes represent untranslated regions

relatedness between twins and yields unbiased standard errors and $p$ values. Analyses were done separately for each of the SNPs and followed up by haplotype analyses. For individual SNP association analyses, we first performed an overall test of genotypic association with two degrees of freedom. In the presence of a significant association, additive, dominant and recessive models (all with one degree of freedom) were further tested to find the best mode of inheritance. Age and menopausal status were included as covariates in the models. Details of our method of testing the association of statistically inferred haplotypes with continuous traits have been described previously [28]. In short, we adapted the haplotype trend regression (HTR) of Zaykin et al. [29] for the analysis of related subjects such as twins by replacing the linear regression with the GEE procedure. Assuming additive effects of the haplotypes on the trait, the HTR tests for the contribution of individual haplotypes. The most frequent haplotype was used as the baseline with which the effects of the other haplotype were contrasted. Haplotypes with estimated frequencies below $5 \%$ in all the subjects were pooled and included in the model as one term. The probabilities of haplotype pairs were estimated with PHASE 2.0 software (available from http://www.depts.washington.edu/ventures/UW_Technol ogy/Express_Licenses/PHASEv2.php, last accessed in July 2006) [30]. A diplotype analysis under a codominant model was also tested by using a weighted GEE, the weights being the probability of each possible haplotype pair combination for an individual as estimated by PHASE 2.0. Only the diplotypes with frequency $>5 \%$ were considered for the diplotype analyses. Obesity-related variables included leptin, weight, BMI, waist, total fat mass, total fat percentage, central fat mass and central fat percentage. Factor analysis was used to combine strongly correlated indices of obesity into two measures: one for general obesity (serum leptin, BMI, weight, total fat mass and total fat percentage) and one for central obesity (waist, central fat mass and central fat percentage). To reduce the likelihood of identifying false-positive associations, we performed analyses of individual obesity-related variables only for those SNPs showing significant results for at least one of the combined scores. We initially used two indices of insulin sensitivity, fasting insulin and the insulin sensitivity measure (SiM). SiM is derived from both fasting and 2-h insulin and glucose data [31] and was calculated according to the following formulae: $\mathrm{SiM}=(0.137 \times \mathrm{SiB}+$ $\mathrm{SiH} 2) / 2$, where the insulin sensitivity baseline (fasting) $(\mathrm{SiB})=10^{8} /($ fasting insulin $\times$ fasting glucose $\times$ distribution volume [VD]); insulin sensitivity $2 \mathrm{~h}$ after OGTT $(\mathrm{SiH} 2)=10^{8} /$ (2-h insulin $\times 2$-h glucose $\times \mathrm{VD}$ ), and $\mathrm{VD}=150 \mathrm{ml} / \mathrm{kg} \times$ body weight. Both fasting insulin $(r=0.68)$ and $\operatorname{SiM}(r=0.92)$ are highly correlated with insulin sensitivity in the normal population. SiM is also a good predictor of diabetes, especially in Europids [32]. We subsequently tested fasting and 2-h glucose based on the hypothesis that direct stimulation of the mobilisation of glucose transporter GLUT4 by PI3K [10] could occur independently of metabolic effects initiated via its activation of PKB. The 
program developed by Purcell et al. [33] was used to calculate the genetic power of the study. It provides automated power analysis for quantitative trait loci association tests in sibships. Assuming a sibling correlation of 0.3 , a sample of 840 dizygotic pairs is adequate to detect a locus effect of $0.5 \%$ with $80 \%$ power (and $\alpha=0.05$ ). The present study, involving 940 dizygotic pairs and an additional 423 monozygotic pairs and 52 singletons, provided even greater power.

\section{Results}

Characteristics of subjects in the Twins UK study sample are shown in Table 1. Among the 13 SNPs chosen from the NCBI database, rs8192679, rs10515070, rs895304 and rs3729981 were not polymorphic in our test sample of 94 subjects. Of the remaining nine polymorphic SNPs, all except rs3730089 (Met326Ile in exon 6) and rs706713 (Tyr73Tyr in exon 1) were located in non-coding regions and their genotype frequencies were consistent with HardyWeinberg proportions. The minor allele frequencies (MAFs) and the pairwise $\mathrm{D}^{\prime}$ and $r^{2}$ of these nine SNPs in 94 subjects are shown in Table 2. All the SNPs had MAFs $>0.05$ and strong LD was observed among rs706713, rs706714, rs7713645 and rs7709243 $\left(\mathrm{D}^{\prime}>0.8\right.$ and $p$ values $<0.05)$. However, except for rs706713 and rs706714 $\left(r^{2}=0.878\right)$, low $r^{2}$ values were found throughout the gene, indicating that in this case tagging would not increase efficiency. This was confirmed by the result that a total of eight tSNPs were selected to represent the nine SNPs by the htSNP2 software with the criterion $R_{\mathrm{L}}^{2} \geq 0.80$ (Table 2). The eight tSNPs rs706713, rs7713645, rs7709243, rs251406, rs40318, rs1550805, rs831125 and rs3730089 were further genotyped in all 2,778 subjects of the Twins UK cohort. ESM Table 3 shows the genotype and allele frequencies of the eight tSNPs, based on one monozygotic twin and both dizygotic twins genotyped for each pair. None of the loci showed deviation from Hardy-Weinberg equilibrium.

To reduce the potential generation of false-positive results through multiple testing, we first analysed the combined central and general obesity scores. We observed significant associations for three tSNPs in at least one of these combined scores (rs7713645, $p=0.072 / 0.040$; rs 7709243, $p=0.033 / 0.020$; rs1550805, $p=0.006 / 0.009$ for central/general obesity scores). These SNPs explained between 0.22 and $0.31 \%$ of the variance in central obesity and between 0.12 and $0.25 \%$ of the variance in general obesity. Since we did not observe any significant effects of rs251406, rs40318, rs706713, rs831125 and rs3730089 on these combined scores (or on any of the glucose or insulin variables), results for these five tSNPs are not further shown. Association results of the three significant tSNPs with the individual obesity phenotypes are shown in Table 3. Carriers of the minor allele of rs 1550805 had significantly higher serum leptin, BMI, weight, total fat, total fat percentage, waist circumference, central fat and central fat percentage, based on additive and dominant models. Carriers of the minor allele of rs7709243 had significantly higher BMI, weight, total fat and central fat. Carriers of the minor allele of rs 7713645 had significantly higher BMI. Carriage of the minor allele of either rs7713645 or rs7709243 was also associated with significantly higher fasting glucose $(0.29-0.42 \%$ of the variance) and glucose $2 \mathrm{~h}$ after the OGTT $(0.6-0.75 \%$ of the variance). However, we did not observe any significant effects of these tSNPs on the two insulin sensitivity indices, fasting insulin and SiM. Association results for homeostasis model assessment, another index of insulin sensitivity, based on fasting levels of insulin and glucose, are not shown because they were identical to those for fasting insulin.

The estimated haplotype frequencies of the eight tSNPs are shown in ESM Table 4. The low LD across this gene resulted in 24 haplotypes with frequency $>1 \%$ and only five of them had frequency $\geq 5 \%$. Based on simulations, Lake et al. [34] suggest that haplotype frequencies need to be at least $5 \%$ to avoid biased regression parameters. The fact that these five haplotypes cover only $50 \%$ of the haplotype diversity made the haplotype analysis based on the eight tSNPs uninformative and difficult to interpret. We decided to focus on the three SNPs which were individually associated with phenotypes to clarify the combined effect of these SNPs. ESM Table 5 shows estimated frequencies of haplotypes constructed by the three SNPs rs7713645, rs7709243 and rs 1550805 . Table 4 shows tests of association including the three common ( $>5 \%)$ haplotypes (111, 221 and 222). Haplotype analyses showed results similar to those for the individual SNP associations, with haplotype rs7713645-rs7709243-rs1550805 222 (frequency 7.2\%) showing higher serum leptin $(p=0.007)$ and higher levels of all body fat measures ( $p$ values $\leq 0.001)$ compared with the most common haplotype, 111 (frequency $45.5 \%$ ). Haplotype 221 (frequency 38.7\%) showed higher fasting glucose $(p=0.035)$ and 2-h glucose $(p=0.021)$ compared with the most common haplotype. SNP rs1550805 had a strong association with obesity variables, whilst the effects on glucose appeared to be associated with rs 7713645 and rs7709243. Because rs7713645 and rs7709243 showed the same alleles among the three most common haplotypes, a more parsimonious haplotype analysis based on one of these two SNPs and rs1550805 was further tested. Results remained unchanged. The 2344 individuals used to reconstruct haplotypes included some subjects with incomplete genotype data. We repeated the haplotype analyses based on the subjects with data on all three SNPs and observed 
Table 2 Pairwise linkage disequilibrium coefficients between the nine SNPs genotyped in 94 subjects

\begin{tabular}{|c|c|c|c|c|c|c|c|c|c|c|}
\hline$\left|\mathrm{D}^{\prime}\right| / r^{2}$ & MAF & rs706713 & rs 706714 & rs 7713645 & rs 7709243 & rs251406 & rs40318 & rs 1550805 & rs831125 & rs3730089 \\
\hline rs 706713 & 0.180 & & $0.878^{\mathrm{a}}$ & $0.183^{\mathrm{a}}$ & $0.171^{\mathrm{a}}$ & 0.017 & 0.017 & 0.003 & 0.001 & 0.000 \\
\hline rs706714 & 0.197 & $1.000^{\mathrm{a}}$ & & $0.213^{\mathrm{a}}$ & $0.186^{\mathrm{a}}$ & 0.015 & 0.040 & 0.008 & 0.003 & 0.000 \\
\hline rs 7713645 & 0.445 & $1.000^{\mathrm{a}}$ & $1.000^{\mathrm{a}}$ & & $0.452^{\mathrm{a}}$ & 0.000 & $0.053^{\mathrm{a}}$ & 0.014 & 0.028 & 0.016 \\
\hline rs 7709243 & 0.483 & $0.808^{\mathrm{a}}$ & $0.828^{\mathrm{a}}$ & $0.808^{\mathrm{a}}$ & & 0.001 & $0.042^{\mathrm{a}}$ & 0.026 & 0.006 & 0.019 \\
\hline rs251406 & 0.331 & 0.416 & 0.357 & 0.026 & 0.048 & & $0.030^{\mathrm{a}}$ & 0.024 & 0.030 & 0.001 \\
\hline rs 40318 & 0.065 & 0.214 & 0.374 & $1.000^{\mathrm{a}}$ & $0.717^{\mathrm{a}}$ & $1.000^{\mathrm{a}}$ & & 0.004 & 0.032 & 0.022 \\
\hline rs1550805 & 0.093 & 0.073 & 0.140 & 0.378 & 0.572 & 0.325 & 1.000 & & 0.002 & 0.002 \\
\hline rs831125 & 0.131 & 0.150 & 0.266 & 0.365 & 0.221 & 0.632 & 0.296 & 0.369 & & 0.025 \\
\hline rs3730089 & 0.149 & 0.014 & 0.009 & 0.324 & 0.304 & 0.080 & 0.230 & 0.055 & 0.909 & \\
\hline$R_{\mathrm{L}}^{2}$ & - & - & 0.903 & - & - & - & - & - & - & - \\
\hline
\end{tabular}

$\left|\mathrm{D}^{\prime}\right|$ below diagonal and $r^{2}$ above diagonal

MAF Minor allele frequency; $R_{\mathrm{L}}{ }^{2}$ proportion of the variance of each non-tagging SNP explained by regression on the tSNPs [21]

${ }^{\mathrm{a}} p<0.05$

the same results as in the analyses based on subjects with data on at least one SNP (data not shown). Diplotype analyses confirmed the results of haplotype analyses: haplotype 222 heterozygotes showed higher levels of body fat measures and haplotype 221 heterozygotes and homozygotes showed higher 2-h glucose (ESM Table 6).

The overall significance of haplotype associations with leptin, BMI, waist and all fat measurements was high and the effect size was relatively large. For example, individuals homozygous for haplotype 222 on average were $5 \mathrm{~kg}$ heavier, had $4 \mathrm{~cm}$ larger waist circumference and $1.73 \mathrm{~kg} / \mathrm{m}^{2}$ greater BMI than those homozygous for 111. However, the percentages of variance in obesity phenotypes explained by PIK3RI haplotypes ranged from only $0.35 \%(p=0.003)$ for central fat percentage to $0.54 \%$ for BMI $(p=0.003)$, which is a reflection of the low frequency of haplotype $222(7.2 \%)$ but much as expected for a trait influenced by many genes.

Table 3 Association analyses of PIK3RI tSNPs rs7713645, rs7709243 and rs1550805

\begin{tabular}{|c|c|c|c|c|c|}
\hline & \multirow{2}{*}{$\begin{array}{l}\text { Number } \\
11 / 12 / 22\end{array}$} & \multicolumn{3}{|l|}{ Mean \pm SD } & \multirow[t]{2}{*}{$p$ value $^{\mathrm{a}}$} \\
\hline & & 11 & 12 & 22 & \\
\hline \multicolumn{6}{|l|}{ rs7713645 } \\
\hline BMI & $622 / 1,202 / 572$ & $24.5 \pm 4.4$ & $24.8 \pm 4.5$ & $25.1 \pm 4.3$ & 0.020 \\
\hline Fasting glucose & $514 / 995 / 480$ & $4.45 \pm 0.53$ & $4.49 \pm 0.56$ & $4.57 \pm 0.56$ & $0.003^{\mathrm{b}}$ \\
\hline 2-h glucose & $273 / 447 / 268$ & $5.04 \pm 1.08$ & $5.27 \pm 1.13$ & $5.28 \pm 1.12$ & $0.023^{\mathrm{c}}$ \\
\hline \multicolumn{6}{|l|}{ rs 7709243} \\
\hline BMI & $674 / 1,332 / 587$ & $24.4 \pm 4.2$ & $24.6 \pm 4.4$ & $25.1 \pm 4.3$ & 0.029 \\
\hline Weight & $675 / 1,332 / 587$ & $64.6 \pm 11.8$ & $65.1 \pm 11.7$ & $66.2 \pm 11.7$ & $0.026^{\mathrm{d}}$ \\
\hline Total fat & $661 / 1,310 / 582$ & $22.7 \pm 8.7$ & $23.4 \pm 8.8$ & $23.9 \pm 8.6$ & $0.031^{\mathrm{d}}$ \\
\hline Central fat & $656 / 1,299 / 580$ & $1.28 \pm 0.74$ & $1.33 \pm 0.73$ & $1.36 \pm 0.70$ & $0.023^{\mathrm{d}}$ \\
\hline Fasting glucose & $571 / 1,106 / 503$ & $4.46 \pm 0.53$ & $4.47 \pm 0.56$ & $4.56 \pm 0.56$ & $0.001^{\mathrm{b}}$ \\
\hline 2-h glucose & $293 / 508 / 257$ & $5.04 \pm 1.09$ & $5.28 \pm 1.11$ & $5.31 \pm 1.19$ & $0.007^{\mathrm{c}}$ \\
\hline \multicolumn{6}{|l|}{ rs 1550805} \\
\hline Leptin & $1,972 / 423 / 11$ & $16.3 \pm 11.9$ & $17.7 \pm 12.6$ & $19.6 \pm 12.3$ & $0.028^{\mathrm{c}}$ \\
\hline BMI & $1,957 / 423 / 11$ & $24.7 \pm 4.4$ & $25.1 \pm 4.6$ & $27.4 \pm 7.0$ & $0.025^{\mathrm{d}}$ \\
\hline Weight & $1,958 / 423 / 11$ & $65.1 \pm 11.8$ & $66.3 \pm 12.2$ & $72.8 \pm 21.3$ & $0.019^{\mathrm{d}}$ \\
\hline Total fat & $1,924 / 420 / 11$ & $23.3 \pm 8.7$ & $24.3 \pm 9.4$ & $28.6 \pm 13.7$ & $0.004^{\mathrm{d}}$ \\
\hline$\%$ Total fat & $1,899 / 412 / 11$ & $35.4 \pm 8.0$ & $36.4 \pm 8.0$ & $39.7 \pm 6.9$ & $0.002^{\mathrm{d}}$ \\
\hline Waist circumference & $1,916 / 420 / 11$ & $78.2 \pm 10.2$ & $79.1 \pm 10.3$ & $82.9 \pm 13.9$ & $0.025^{\mathrm{d}}$ \\
\hline Central fat & $1,912 / 416 / 11$ & $1.32 \pm 0.73$ & $1.39 \pm 0.74$ & $1.65 \pm 1.01$ & $0.005^{\mathrm{d}}$ \\
\hline$\%$ Central fat & $1,912 / 416 / 11$ & $31.1 \pm 11.5$ & $32.1 \pm 11.5$ & $34.5 \pm 11.0$ & $0.005^{\mathrm{c}}$ \\
\hline
\end{tabular}

${ }^{a} p$ value adjusted for age

${ }^{\mathrm{b}} p$ value under the recessive genetic model

${ }^{c} p$ value under the dominant genetic model

${ }^{\mathrm{d}} p$ value under the additive genetic model 
Table 4 Tests of association of tSNP haplotypes (frequency $>5 \%$ ) with phenotypes

\begin{tabular}{|c|c|c|c|c|c|c|}
\hline \multirow[t]{2}{*}{ Variable } & \multicolumn{2}{|l|}{ Haplotype $221^{\text {a }}$} & \multicolumn{2}{|l|}{ Haplotype $222^{\mathrm{a}}$} & \multicolumn{2}{|l|}{ Overall } \\
\hline & $\beta$ & $p^{\mathrm{b}}$ & $\beta$ & $p^{\mathrm{b}}$ & Explained variance $(\%)$ & $p^{\mathrm{b}}$ \\
\hline Leptin & 0.46 & NS & $4.67(1.68-7.65)$ & 0.007 & 0.43 & 0.043 \\
\hline BMI & 0.38 & NS & $1.78(0.71-2.85)$ & 0.001 & 0.54 & 0.003 \\
\hline Weight & 0.83 & NS & $5.00(2.07-7.93)$ & 0.001 & 0.47 & 0.003 \\
\hline Total fat & 0.43 & NS & $4.00(1.90-6.11)$ & 0.0004 & 0.43 & 0.002 \\
\hline$\%$ Total fat & 0.24 & NS & $3.30(1.47-5.13)$ & 0.0004 & 0.44 & 0.003 \\
\hline Waist & 0.02 & NS & $4.00(1.69-6.62)$ & 0.001 & 0.41 & 0.005 \\
\hline Central fat & 0.01 & NS & $0.28(0.11-0.45)$ & 0.001 & 0.44 & 0.002 \\
\hline$\%$ Central fat & 0.28 & NS & $4.62(2.00-7.23)$ & 0.001 & 0.35 & 0.003 \\
\hline Fasting glucose & $0.08(0.01-0.15)$ & 0.035 & 0.03 & NS & 0.36 & NS \\
\hline 2-h glucose & $0.24(0.03-0.45)$ & 0.021 & 0.31 & NS & 1.01 & 0.02 \\
\hline
\end{tabular}

${ }^{\text {a }}$ Estimates are contrasts with the most common haplotype, 111, where 1 denotes the major allele and 2 denotes the minor allele of the SNPs rs 7713645 , rs7709243 and rs 1550805

${ }^{\mathrm{b}} p$ value adjusted for age

\section{Discussion}

We have examined the association of a set of eight tSNPs spanning the PIK3Rl gene with a range of phenotypes representing serum leptin, anthropometry, body fat and glucose/insulin homeostasis in a large population of Europid British female twins. We showed in single-tSNP analyses that one tSNP in particular, rs1550805, was significantly associated with serum leptin, BMI, weight, waist circumference and all the fat variables. tSNPs rs7713645 and rs7709243 were significantly associated with BMI, rs7709243 with weight, total and central fat, and both tSNPs with fasting glucose and glucose $2 \mathrm{~h}$ after an OGTT. Haplotypic analyses of these three tSNPs confirmed the significance of the individual associations, haplotype 222 showing significantly higher levels of serum leptin and all body fat measures. Haplotype 221 was significantly associated with higher levels of fasting glucose and 2$\mathrm{h}$ glucose, but the most significant finding is the association of rs 1550805 with serum leptin and body fat measurements.

The main strength of our study lies in the large size of the study sample, the availability of detailed measurements of body fat by dual X-ray absorptiometry in all subjects, and comprehensive measurement of variation in the gene. This is the first gene-wide association study of PIK3RI and the first reported association of variants with leptin and body fat measurements, strengthening the candidature of the gene in susceptibility to obesity and insulin resistance. Assuming a sibling correlation of 0.3 , the present study provided power in excess of $80 \%$ (and $\alpha=0.05$ ) to detect a locus effect of $0.5 \%$ [33]. It should be noted that we have established these associations only in a female twin population, but that our findings in the twin subjects can be considered as representative of the UK female popula- tion as a whole [24]. We have previously found few differences between twins and singletons in the population generally, the only indication being that monozygotic twins have a slightly lower weight and a smaller variance for weight than dizygotic twins and singletons [24].

The comprehensiveness of coverage of the PIK3RI gene by our selected tSNPs was limited by the availability of SNPs on public databases at the time of the study. Information from the HapMap (http://www.hapmap.org) for PIK3R1 has only recently become available. Seven of our eight tSNPs are within the 27 common SNPs (minor allele frequency $\geq 5 \%$ in Centre d'Etude du Polymorphisme Humain [CEPH] subjects) posted on the current release of HapMap (Phase II, January 2006). Sixteen HapMap SNPs cannot be tagged by our selection. The gene lies in a region of low LD, shown by the low $r^{2}$ values found throughout the gene, so that eight tSNPs were needed to represent the nine validated SNPs available in public databases at the time. All of our associated tSNPs, with the exception of the Met326Ile variant rs3730089, are intronic and are most likely to be LD markers.

Previously reported investigations of variation in PIK3R1 have centred on tests of association with type 2 diabetes and associated phenotypes [16, 18, 35, 36], on account of the well-known involvement of PI3K in insulin signalling in peripheral tissues [37] and the hypothalamus [2]. Relatively little attention has been paid to the influence of PI3K on fat deposition, which could occur through the antilipolytic effect of insulin and be influenced by leptin through cross-talk with the insulin-signalling path $[2,38]$. Aside from the Met326Ile variant [16] and an IVS4+82 SNP [19], there are to our knowledge no other reported studies of association of other PIK3RI variants with variables characterising the action of leptin or insulin. 
We found no significant associations between any single tSNPs or haplotypes with fasting insulin and the SiM measure of insulin sensitivity. Among our tested tSNPs, the Met326Ile variant rs3730089 was previously reported to be associated with insulin sensitivity in 380 young healthy Danish subjects [16]. However, this group failed to replicate the association in a later study of 1,190 individuals [18].

In summary, we have established an association between PIK3R1 SNP rs1550805 with serum leptin and a range of body fat measures in a large Europid female twin population. Phosphorylation of IRS2 by JAK2 lies at a convergence point of the leptin and insulin signalling pathways [39]. Leptin potentiates insulin signalling through its activation of IRS2 by JAK2 phosphorylated at the leptin receptor [40] and activates PI3K in muscle via this IRS2dependent pathway [41]. The importance of PI3K signalling via IRS1 in the lipid-lowering effect of leptin in liver has been shown by Huang et al. [9], and Niswender et al. [3] have shown that PI3K and IRS2 are involved in the anorexic response to leptin in the hypothalamus. We speculate that the site marked by rs 1550805 may reflect diminished ability of PI3K to signal via IRS1 or IRS2 in response to leptin. This could lead to impaired leptin action, failure of the normal feedback regulation of leptin levels, and the accumulation of body fat.

PI3K regulatory subunit isoforms p85 $\alpha, \mathrm{p} 55 \alpha$ and p50 $\alpha$, generated from the same PIK3R1 gene by alternative splicing [11], negatively modulate catalytic activity to different extents [12], the strongest effect being shown by p $85 \alpha$ [13]. Future investigations need to focus on searching for potentially functional SNPs in the isoform-specific exons as well as putative regulatory regions in $P I K 3 R 1$, in view of evidence that the balance and activity of the isoforms affect the overall activity of the PI3K enzyme [12, 13, 42, 43].

Acknowledgements This study was funded by the Wellcome Trust Project grant No. 073142. The Twin Research and Genetic Epidemiology Unit and Twins UK received support from the Wellcome Trust, the Arthritis Research Campaign, the Chronic Disease Research Foundation and the European Union 5th Framework Programme Genom EU twin no. QLG2-CT-2002-01254. This research was partly conducted within the network of the London IDEAS Genetic Knowledge Park, utilising the St George's, University of London, Medical Biomics Centre.

\section{References}

1. Schwartz MW, Woods SC, Porte D, Seeley RJ, Baskin DG (2000) Central nervous system control of food intake. Nature 404:661-671

2. Niswender KD, Morrison CD, Clegg DJ et al (2003) Insulin activation of phosphatidylinositol 3-kinase in the hypothalamic arcuate nucleus: a key mediator of insulin-induced anorexia. Diabetes 52:227-231
3. Niswender KD, Morton GJ, Stearns WH, Rhodes CJ, Myers MG, Schwartz MW (2001) Intracellular signalling. Key enzyme in leptin-induced anorexia. Nature 413:794-795

4. Kim YB, Uotani S, Pierroz DD, Flier JS, Kahn BB (2000) In vivo administration of leptin activates signal transduction directly in insulin-sensitive tissues: overlapping but distinct pathways from insulin. Endocrinology 141:2328-2339

5. Carvalheira JB, Ribeiro EB, Folli F, Velloso LA, Saad MJ (2003) Interaction between leptin and insulin signaling pathways differentially affects JAK-STAT and PI 3-kinase-mediated signaling in rat liver. Biol Chem 384:151-159

6. Benomar Y, Roy AF, Aubourg A, Djiane J, Taouis M (2005) Cross down-regulation of leptin and insulin receptor expression and signalling in a human neuronal cell line. Biochem $\mathrm{J}$ 388:929-939

7. Considine RV, Sinha MK, Heiman ML et al (1996) Serum immunoreactive-leptin concentrations in normal weight and obese humans. N Engl J Med 334:292-295

8. Zhao AZ, Huan JN, Gupta S, Pal R, Sahu A (2002) A phosphatidylinositol 3-kinase phosphodiesterase 3B-cyclic AMP pathway in hypothalamic action of leptin on feeding. Nat Neurosci 5:727-728

9. Huang W, Dedousis N, Bhatt BA, O'Doherty RM (2004) Impaired activation of phosphatidylinositol 3-kinase by leptin is a novel mechanism of hepatic leptin resistance in diet-induced obesity. J Biol Chem 279:21695-21700

10. Holman GD, Kasuga M (1997) From receptor to transporter: insulin signalling to glucose transport. Diabetologia 40:991-1003

11. Fruman DA, Cantley LC, Carpenter CL (1996) Structural organization and alternative splicing of the murine phosphoinositide 3-kinase p85 alpha gene. Genomics 37:113-121

12. Ueki K, Algenstaedt P, Mauvais-Jarvis F, Kahn CR (2000) Positive and negative regulation of phosphoinositide 3-kinasedependent signaling pathways by three different gene products of the p85alpha regulatory subunit. Mol Cell Biol 20:8035-8046

13. Terauchi Y, Tsuji Y, Satoh S et al (1999) Increased insulin sensitivity and hypoglycaemia in mice lacking the p85 alpha subunit of phosphoinositide 3-kinase. Nat Genet 21:230-235

14. Shepherd PR, Withers DJ, Siddle K (1998) Phosphoinositide 3kinase: the key switch mechanism in insulin signalling. Biochem $\mathrm{J}$ 333:471-490

15. Inukai K, Funaki M, Ogihara T et al (1997) p85alpha gene generates three isoforms of regulatory subunit for phosphatidylinositol 3-kinase (PI 3-kinase), p50alpha, p55alpha, and p85alpha, with different PI 3-kinase activity elevating responses to insulin. J Biol Chem 272:7873-7882

16. Hansen T, Andersen CB, Echwald SM, Urhammer SA, Clausen JO, Vestergaard $\mathrm{H}$ et al (1997) Identification of a common amino acid polymorphism in the p85alpha regulatory subunit of phosphatidylinositol 3-kinase: effects on glucose disappearance constant, glucose effectiveness, and the insulin sensitivity index. Diabetes 46:494-501

17. Baynes K, Beeton CA, Panayotou G et al (2000) Natural variants of human p85 alpha phosphoinositide 3-kinase in severe insulin resistance: a novel variant with impaired insulin-stimulated lipid kinase activity. Diabetologia 43:321-331

18. Hansen L, Zethelius B, Berglund L et al (2001) In vitro and in vivo studies of a naturally occurring variant of the human p85alpha regulatory subunit of the phosphoinositide 3-kinase: inhibition of protein kinase $\mathrm{B}$ and relationships with type 2 diabetes, insulin secretion, glucose disappearance constant, and insulin sensitivity. Diabetes 50:690-693

19. Barroso I, Luan J, Middelberg RP et al (2003) Candidate gene association study in type 2 diabetes indicates a role for genes involved in beta-cell function as well as insulin action. PLoS Biol 1:E20 
20. Neale BM, Sham PC (2004) The future of association studies: gene-based analysis and replication. Am J Hum Genet 75: 353-362

21. Chapman JM, Cooper JD, Todd JA, Clayton DG (2003) Detecting disease associations due to linkage disequilibrium using haplotype tags: a class of tests and the determinants of statistical power. Hum Hered 56:18-31

22. Stram DO, Haiman CA, Hirschhorn JN et al (2003) Choosing haplotype-tagging SNPS based on unphased genotype data using a preliminary sample of unrelated subjects with an example from the Multiethnic Cohort Study. Hum Hered 55:27-36

23. Spector TD, MacGregor AJ (2002) The St. Thomas' UK Adult Twin Registry. Twin Res 5:440-443

24. Andrew T, Hart D, Snieder H, de Lange M, Spector TD, MacGregor AJ (2001) Are twins and singletons comparable? A study of disease-related and lifestyle characteristics in adult women. Twin Res 4:464-477

25. Thompson D, Stram D, Goldgar D, Witte JS (2003) Haplotype tagging single nucleotide polymorphisms and association studies. Hum Hered 56:48-55

26. Abecasis GR, Cookson WO (2000) GOLD - graphical overview of linkage disequilibrium. Bioinformatics 16:182-183

27. Trégouët D-A, Ducimetère P, Tiret L (1997) Testing association between candidate-gene markers and phenotype in related individuals, by use of estimating equations. Am J Hum Genet 61:189-199

28. Dong Y, Zhu H, Wang X et al (2004) Obesity reveals an association between blood pressure and the G-protein beta3subunit gene: a study of female dizygotic twins. Pharmacogenetics $14: 419-427$

29. Zaykin DV, Westfall PH, Young SS, Karnoub MA, Wagner MJ, Ehm MG (2002) Testing association of statistically inferred haplotypes with discrete and continuous traits in samples of unrelated individuals. Hum Hered 53:79-91

30. Stephens M, Donnelly P (2003) A comparison of bayesian methods for haplotype reconstruction from population genotype data. Am J Hum Genet 73:1162-1169

31. Avignon A, Boegner C, Mariano-Goulart D, Colette C, Monnier L (1999) Assessment of insulin sensitivity from plasma insulin and glucose in the fasting or post oral glucose-load state. Int J Obes Relat Metab Disord 23:512-517

32. Hanley AJ, Williams K, Gonzalez C et al (2003) Prediction of type 2 diabetes using simple measures of insulin resistance: combined results from the San Antonio Heart Study, the Mexico City Diabetes Study, and the Insulin Resistance Atherosclerosis Study. Diabetes 52:463-469

33. Purcell S, Cherny SS, Sham PC (2003) Genetic Power Calculator: design of linkage and association genetic mapping studies of complex traits. Bioinformatics 19:149-150

34. Lake SL, Lyon H, Tantisira K et al (2003) Estimation and tests of haplotype-environment interaction when linkage phase is ambiguous. Hum Hered 55:56-65

35. Baier LJ, Wiedrich C, Hanson RL, Bogardus C (1998) Variant in the regulatory subunit of phosphatidylinositol 3-kinase (p85alpha): preliminary evidence indicates a potential role of this variant in the acute insulin response and type 2 diabetes in Pima women. Diabetes 47:973-975

36. Almind K, Delahaye L, Hansen T, Van Obberghen E, Pedersen O, Kahn CR (2002) Characterization of the Met326Ile variant of phosphatidylinositol 3-kinase p85alpha. Proc Natl Acad Sci USA 99:2124-2148

37. Shepherd PR (2005) Mechanisms regulating phosphoinositide 3kinase signalling in insulin-sensitive tissues. Acta Physiol Scand $183: 3-12$

38. Niswender KD, Schwartz MW (2003) Insulin and leptin revisited: adiposity signals with overlapping physiological and intracellular signaling capabilities. Front Neuroendocrinol 24:1-10

39. Kubota N, Terauchi Y, Tobe K et al (2004) Insulin receptor substrate 2 plays a crucial role in beta cells and the hypothalamus. J Clin Invest 114:917-927

40. Yaspelkis BB 3rd, Davis JR, Saberi M et al (2001) Leptin administration improves skeletal muscle insulin responsiveness in diet-induced insulin-resistant rats. Am J Physiol Endocrinol Metab 280:E130-E142

41. Kellerer M, Koch M, Metzinger E, Mushack J, Capp E, Haring, HU (1997) Leptin activates PI-3 kinase in C2C12 myotubes via janus kinase-2 (JAK-2) and insulin receptor substrate-2 (IRS-2) dependent pathways. Diabetologia 40:1358-1362

42. Chen D, Mauvais-Jarvis F, Bluher M et al (2004) p50alpha/ p55alpha phosphoinositide 3-kinase knockout mice exhibit enhanced insulin sensitivity. Mol Cell Biol 24:320-329

43. Kerouz, NJ, Horsch D, Pons S, Kahn CR (1997) Differential regulation of insulin receptor substrates-1 and -2 (IRS-1 and IRS-2) and phosphatidylinositol 3-kinase isoforms in liver and muscle of the obese diabetic $(o b / o b)$ mouse. J Clin Invest 100:3164-3172 